\title{
HSPH1 T17 Repeat
}

National Cancer Institute

\section{Source}

National Cancer Institute. HSPH1 T17 Repeat. NCI Thesaurus. Code C116969.

A mononucleotide DNA repeat consisting of 17 thymine bases, which is found within intron 8 of the human HSPH1 gene. Variation in, or somatic mutation of this region causes the expression of an aberrant HSPH1 mRNA transcript that is missing exon 9 (HSPH1-deltaE9), which encodes a protein that lacks the HSPH1 substrate binding domain. 